\title{
A Clinico-Demographic Profile of Atrial Fibrillation - A Hospital Based Descriptive Study from South India
}

\author{
Yerragunda Uday Kuamr ${ }^{1}$, Barkur Ananthkrishna Shastry², Ganesh V. Shetty ${ }^{3}$, Sharath P. Madhyastha ${ }^{4}$ \\ 1,2,3,4 Department of Medicine, Kasturba Medical College, Manipal Academy of Higher Education, \\ Manipal, Karnataka, India.
}

\section{ABSTRACT}

\section{BACKGROUND}

Atrial fibrillation (AF) is the commonest arrhythmia seen in clinical practice. As it is known to contribute to significant mortality and morbidity, it is regarded as a major public health problem. There are various predisposing factors for AF such as hypertension, rheumatic heart disease, diabetes mellitus among many others. The aim of this study was to evaluate the clinical profile and echocardiographic features in patients of $\mathrm{AF}$.

\section{METHODS}

A total of 103 patients aged above 18 years and recently diagnosed with AF were enrolled in the study. These patients were subjected to a detailed general physical and systemic examination. Relevant blood investigations, chest X-ray, electrocardiography, and echocardiography were done in all patients. Various clinical and investigational data were recorded, and results were tabulated.

\section{RESULTS}

The mean age of the study group was $63.5 \pm 14.09$ years with a male preponderance. AF secondary to a non-valvular aetiology (76.7 \%) formed the majority. Hypertension was the most common cause overall contributing to $29.12 \%$ followed by rheumatic heart disease (23.3\%). A total of 33 patients exhibited LA size greater than $5 \mathrm{cms}$ and LA clot was observed in $4 \%$ of patients on transthoracic echocardiography.

\section{CONCLUSIONS}

Hypertension is observed to be a major risk factor for AF and hence it needs to be detected and treated early. Improving the lifestyle habits such as diet and exercise can help in reducing the prevalence of hypertension and thus the risk of developing $\mathrm{AF}$.

\section{KEY WORDS}

Atrial Fibrillation, Echocardiography, Arrhythmias
Corresponding Author: Dr. Ganesh V. Shetty, Assistant Professor, Department of Medicine, Kasturba Medical College, Manipal Academy of Higher Education, Manipal - 576104, Karnataka, India.

E-mail:ganeshetty@gmail.com

DOI: 10.14260/jemds/2020/779

How to Cite This Article:

Kumar YU, Shastry BA, Shetty GV, et al. A clinico-demographic profile of atrial fibrillation - a hospital based descriptive study from South India. J Evolution Med Dent Sci 2020;9(47):3551-3555, DOI: 10.14260/jemds/2020/779

Submission 27-08-2020,

Peer Review 08-10-2020,

Acceptance 13-10-2020,

Published 23-11-2020.

Copyright (C) 2020 Yerragunda Uday Kumar et al. This is an open access article distributed under Creative Commons Attribution License [Attribution 4.0 International (CC BY 4.0)] 


\section{BACKGROUND}

Atrial fibrillation presents with an uncoordinated, rapid, and irregular atrial activation with loss of atrial contraction and an irregular ventricular rate. The ventricular rate tends to be rapid and variable in amplitude, shape and timing with a rate of $300-600$ beats per minute. However, it is observed that patients with high vagal tone or AV (Atrio-Ventricular) nodal conduction disease may have slower ventricular rates.1,2

$\mathrm{AF}$ is found to be the commonest sustained arrhythmia and a major public health problem. In addition, it is a common arrhythmia requiring frequent hospitalization. ${ }^{3}$ The overall prevalence rate of $\mathrm{AF}$ in general population is found to be 0.4 $1 \%{ }^{4}$ The prevalence of AF is known to increase with age, with more than $95 \%$ of AF patients being above 60 years of age. It is estimated that lifetime risk of developing $\mathrm{AF}$ for men at 40 years of age is approximately $25 \%$. AF tends to afflict men more than women and whites more than the blacks. ${ }^{1}$ Underlying heart disorders such as coronary heart disease, valvular heart disease, left ventricular dysfunction, hypertrophic cardiomyopathy and congenital heart disease are some of the well-established risk factors for $\mathrm{AF}^{3}{ }^{3}$ The risk factors other than age and underlying cardiac disease for developing AF include hypertension, diabetes mellitus, obesity, and sleep apnea. ${ }^{1}$

The patients with AF can present with varied clinical features such as dyspnoea, palpitations, chest pain and syncope. The key factors responsible for the pathogenesis of $\mathrm{AF}$ include calcium handling abnormalities, structural remodelling, ion channel dysfunction and autonomic neural dysregulation. It is observed that AF can result in significant health consequences in the form of frequent hospital visits, impaired quality of life and increased medical expenses. ${ }^{3}$ Additionally, it can also result in complications such as heart failure and stroke. It is observed that patients with AF have an increased risk of developing heart failure and alternatively patients with heart failure have an increased risk of developing AF. The risk of stroke is increased by fivefold in patients with $\mathrm{AF}$ and $\mathrm{AF}$ is estimated to be the cause in $25 \%$ of strokes. Dementia and silent strokes are more frequently seen in patients of AF. Some of the other risk factors of AF include Chronic Obstructive Pulmonary Disease (COPD), hyperthyroidism, smoking, alcohol intake, and drugs such as theophylline. ${ }^{2}$ Estimation of Left Atrial (LA) size on echocardiography plays a significant role in $\mathrm{AF}$ as it is a marker of left ventricular dysfunction and the degree of increase in LA pressure. $^{3}$ In view of multiple aetiologies, varying presentations and echocardiographic features, the present study was undertaken to study the clinical profile and the utility of echocardiography (ECHO) in AF.

\section{METHODS}

The present study was a hospital based descriptive study, enrolling a total of 103 patients with $\mathrm{AF}$, and conducted over a period of 3 years from 2016 to 2018. Sample size was taken based on the convenience of the study. All the patients who presented with features suggestive of $\mathrm{AF}$ and were 18 years or older were enrolled in the study. A diagnosis of AF was confirmed in them based on the typical electrocardiographic (ECG) changes. Those patients wherein a diagnosis of AF was already established and / or were on treatment for the same were excluded from the study. The above study was approved by the Institutional Ethics Committee and a written informed consent was taken from all the participants.

A detailed history pertaining to the chief complaints that the patients presented with such as dyspnoea, chest pain, palpitations and fatigue were obtained. An enquiry was made into the associated co-morbid conditions and personal habits of patients. All patients were subjected to an elaborate general and systemic examination. The cardiovascular system examination included few detailed assessments such as inspection, palpation, and auscultation of cardiovascular system, presence of murmurs, looking for signs of cardiac failure and Pulmonary Artery Hypertension (PAH), and the presence of cardiomegaly. All patients underwent routine investigations such as CBC (Complete Blood Count), RFT (Renal Function Tests), LFT (Liver Function Tests), CXR (Chest X-Ray), TSH (Thyroid Stimulating Hormone) and ECG. AF was diagnosed based on the ECG findings of absence of P waves, irregular R-R intervals with a rapid ventricular rate.

A 2D-Echocardiogram (2D-ECHO) was done in all ECG proven AF patients, to know LA (Left Atrial) and LV (Left Ventricular) dimensions; assess the LV wall thickness and function as well as to recognize the underlying pathological conditions so as to know the aetiology of $\mathrm{AF}$ (valvular, myocardial or pericardial aetiology). All patients were evaluated using M Mode and two-dimensional transthoracic echocardiography and colour flow Doppler examination using Philips Sonos. Each 2D ECHO consisted of parasternal long and short axes, and apical two and four chamber views. The conventions of American Society of Echocardiography were followed in obtaining LA dimensions, LV end systolic and end diastolic dimensions, as well as right ventricular end diastolic dimensions. The anterior-posterior LA dimension was calculated in the short axis view from M-mode echocardiography and LA area or volume was measured in apical-chamber or four-chamber views. Left ventricular volumes were measured and Ejection Fraction (EF) was calculated.

\section{Statistical Analysis}

The Statistical software SAS 9.2, SPSS 15.0, Stata 10.1, MedCalc 9.0.1, Systat 12.0 and R environment ver. 2.11.1 were used for the analysis of the data and Microsoft Word and Excel have been used to generate graphs and tables.

\section{RESULTS}

\section{Patient Demographics}

A total of 103 patients above 18 years diagnosed with a new onset $\mathrm{AF}$ were included in the study. The mean age of the study group was 63.5 years \pm 14.09 with $62(60 \%)$ males and 41 (40

$\%)$ females. Majority of the patients (40\%) were in the age group of 46 - 65 years. Table 1 illustrates the age distribution of patients with $\mathrm{AF}$. 


\begin{tabular}{|cc|}
\hline Age in Yrs. & No. of Patients \\
\hline $18-45$ & $14(13.59 \%)$ \\
$46-65$ & $41(39.80 \%)$ \\
$66-75$ & $28(27.18 \%)$ \\
$>75$ & $20(19.42 \%)$ \\
\hline Table 1. Age Distribution of Patients with $\boldsymbol{A F}$ \\
\hline
\end{tabular}

\section{Presenting Clinical Features}

Majority of the patients $(\mathrm{n}=64)$ presented with dyspnoea followed by pedal oedema $(n=24)$. The other features with which the patients manifested were fatigue, stroke, palpitation, chest pain, syncope and giddiness. Out of 103 patients, 33 gave a history of smoking and 10 patients had history of alcohol consumption. An elevated Jugular Venous Pulse (JVP) was found in $20 \%$ of cases, an irregularly irregular pulse was observed in $90 \%$ of cases. A palpable P2 was observed in 36 patients. A varying S1 was found in $83 \%$ and a loud P2 was heard in $19 \%$ on auscultation. Pansystolic murmur was heard over both mitral and tricuspid area in 10 and 40 patients, respectively and a mid-diastolic murmur was heard in 15 patients. Presystolic accentuation was noticed in 9 $\%$ of patients. Around $20 \%$ of patients had crepitations on auscultation suggestive of pulmonary oedema. Neurological deficits were detected in $6 \%$, and heart failure in $15 \%$ of patients. The type of AF commonly encountered in the present study was the persistent type $(n=64)$, followed by paroxysmal $(n=24)$ and permanent $(n=15)$.

\begin{tabular}{|cc|}
\hline Aetiology & Number $(\mathbf{n}=\mathbf{7 9})$ \\
Hypertension & 30 \\
COPD & 18 \\
Diabetes Mellitus & 14 \\
IHD & 7 \\
Dilated Cardiomyopathy & 5 \\
Thyrotoxicosis & 4 \\
Lone AF & 1 \\
\hline Table 2. Non-Valvular Causes of $\mathbf{A F}$ \\
\hline
\end{tabular}

\section{Aetiology of AF}

The commonest cause of AF encountered in our study was from non-valvular group contributing to $76.7 \%$ of cases. Hypertension was observed to be the most common nonvalvular cause for AF. The other non-valvular causes of AF is depicted in Table 2. Amongst the valvular aetiology of AF, Rheumatic Heart Disease (RHD) was the commonest cause accounting for 24 out of 103 patients. The valvular involvement in RHD is represented in Table 3.

\begin{tabular}{|cc|}
\hline Valvular Involvement & Number $(\mathbf{n}=\mathbf{2 ~ 4 )}$ \\
MS with TR & 9 \\
MS with MR with TR & 7 \\
Isolated MS & 5 \\
MR & 3 \\
\hline Table 3. Valvular Involvement in RHD \\
\hline
\end{tabular}

\section{Investigation Findings}

The findings on chest X-ray included cardiomegaly (31\%), COPD (22\%), pulmonary oedema (20\%) and $4 \%$ had Acute Respiratory Distress Syndrome (ARDS) due to acute febrile illness and the remaining $26 \%$ revealed no findings on chest X-ray. All 103 patients were found to have absence of $p$ wave with a varying R-R interval. However, 94 patients had additional ECG findings such as non-specific ST-T changes (32 $\%)$, Left Ventricular Hypertrophy (LVH) (20 \%), Right
Ventricular Hypertrophy (RVH) (17\%), Right Bundle Branch Block (RBBB) (10\%), Left Bundle Branch Block (LBBB) (5\%), $\mathrm{AF}$ with aberrancy and block (4\%) and digitalis effects (3\%). The commonest ECHO findings noted in this study were pulmonary artery hypertension (57 \%), tricuspid regurgitation (58\%), RHD (23\%), dilated atria (40\%), systolic dysfunction (9\%), LVH (29\%) and regional wall motion abnormalities suggestive of IHD (Ischemic Heart Disease) (9\%). Left atrial clot was noticed in $4 \%$ of patients. Table 4 depicts the LA size as encountered on 2D ECHO.

\begin{tabular}{|cc|}
\hline LA Size & Number of Patients \\
Less than $4 \mathrm{~cm}$ & 63 \\
$4-5 \mathrm{~cm}$ & 7 \\
More than $5 \mathrm{~cm}$ & 33 \\
\hline Table 4. Table Illustrating the Varying LA Size on 2D-ECHO \\
\hline
\end{tabular}

\section{DISCUSSION}

Atrial fibrillation is the most common arrhythmia encountered in clinical practice characterized by uncoordinated atrial activity resulting in an irregularly irregular ventricular rhythm with a rate of $300-600$ beats per minute. ${ }^{2}$ It has an estimated prevalence of $3 \%$ in adults and its prevalence increased progressively with age. Some of the well-known risk factors of $\mathrm{AF}$ include hypertension, valvular heart disease, male gender, obesity and alcohol consumption. ${ }^{3}$

The mean age of presentation in the present study was found to be 63.5 years with majority of the patients ( $86 \%$ ) above 46 years of age. This was in concordance with the study by Mohan G et al wherein the mean age was $67.02 \pm 12.50$ years with more than $90 \%$ of patients above 50 years of age. ${ }^{2}$ The prevalence of atrial fibrillation increases substantially with age. This was an observed trend in several other studies, the most notable of them being the Rotterdam study and ATRIA study. ${ }^{4,5}$ However, these studies have been limited by relatively small numbers of patients with $\mathrm{AF}$, restriction of age ranges, ethnical homogeneity. . $^{6}$

There was a slight male preponderance in our study similar to other earlier studies. This could be explained by the fact that the prevalence of diabetes, hypertension, COPD, and IHD is more common in men than women, which in turn predisposes them to develop AF. In addition, the degree of shortening of atrial Effective Refractory Period (ERP) was observed to be less in premenopausal women when compared with postmenopausal women and age-matched men, thus suggesting the protective role of oestrogen. ${ }^{10}$

\begin{tabular}{|ccccc|}
\hline & $\begin{array}{c}\text { The } \\
\text { Framingham } \\
\text { Study }\end{array}$ & $\begin{array}{c}\text { Cardiovascular } \\
\text { Health Study }\end{array}$ & $\begin{array}{c}\text { ALFA } \\
\text { Study }\end{array}$ & Present \\
Age (Mean) & 72 & - & 68.7 & 63.5 \\
Smoking & $34.8 \%$ & - & - & $32.03 \%$ \\
DM & $16.3 \%$ & - & $10.7 \%$ & $13.6 \%$ \\
HTN & $44 \%$ & - & $39.4 \%$ & $29.12 \%$ \\
Congestive & $20.6 \%$ & $19 \%$ & $29.8 \%$ & $15 \%$ \\
Heart Failure & $16 \%$ & $4.3 \%$ & $15.2 \%$ & $23.3 \%$ \\
RHD & $25 \%$ & $22 \%$ & $16.6 \%$ & $6.8 \%$ \\
IHD & - & $14.8 \%$ & $8.4 \%$ & $6 \%$ \\
Stroke & - & $2.4 \%$ & $3.1 \%$ & $3.9 \%$ \\
Thyrotoxicosis & $36 \%$ & $7.7 \%$ & $11.2 \%$ & $17.5 \%$ \\
COPD & Table 5. Comparison of Risk Factors and \\
\hline \multicolumn{5}{c}{ Associations of AF with Earlier Studies } \\
\hline
\end{tabular}


The aetiological factors of $\mathrm{AF}$ were categorized into valvular and non-valvular groups. Non-valvular causes formed the majority in the present study (76.7\%) out of which hypertension formed a significant majority (37.9\%). This was similar to the study by Mohan $\mathrm{G}$ et al, however, this finding did not correlate with the study by Singh et al who reported valvular aetiology (RHD) as the cause in $60 \%$ of patients. ${ }^{2,11}$ In the present study, valvular cause in the form of RHD was observed in $23.3 \%$ of patients. Risk factors for developing AF in the Framingham Heart study were hypertension (44\%), diabetes (16\%), prior myocardial infarction (26\%), COPD (36 $\%)$ and valvular heart disease $(17 \%) .{ }^{12}$ Similar findings have been reported in the Cardiovascular Health Study as well.13 Increase in LV thickness and stiffness due to hypertension impairs LV diastolic function which leads to LA stretch and pressure and LA remodelling and dysfunction. Structural, contractile, architectural and electrophysiological changes in atria collectively called atrial cardiomyopathy occurs due to atrial remodelling ultimately leading to $\mathrm{AF} .{ }^{14}$ The other risk factors under non-valvular group include COPD (22.8\%), diabetes mellitus $(17.7 \%)$, IHD $(8.9 \%)$, dilated cardiomyopathy (6.3\%), and thyrotoxicosis (5.1\%). However, in one patient, the aetiology of AF could not be established. Neurological deficits in the form of stroke was observed in $6 \%$ of our patients and $15 \%$ of our patients showed features of heart failure. Table 4 illustrates the comparison of the present study with earlier studies conducted.10,13,15

Majority of our patients (62.1\%) presented with dyspnoea followed by pedal oedema (23.3\%). Similarly, Mohan G et al reported dyspnoea and pedal oedema as the predominant symptoms in $56 \%$ and $39 \%$ of patients, respectively. ${ }^{2}$ However, Shaik Sulaiman Meeran et al reported palpitation and dyspnoea as the predominant symptoms in $52 \%$ and 14 $\%$, respectively. ${ }^{3}$ Similarly, ALFA study, reported palpitations in $54 \%$ of the patients, dyspnoea in $44 \%$, angina in $10 \%$ and syncope and dizzy spells in $10 \% .{ }^{15}$ Palpitations on exertion was observed in $11 \%$ of patients in the present study.

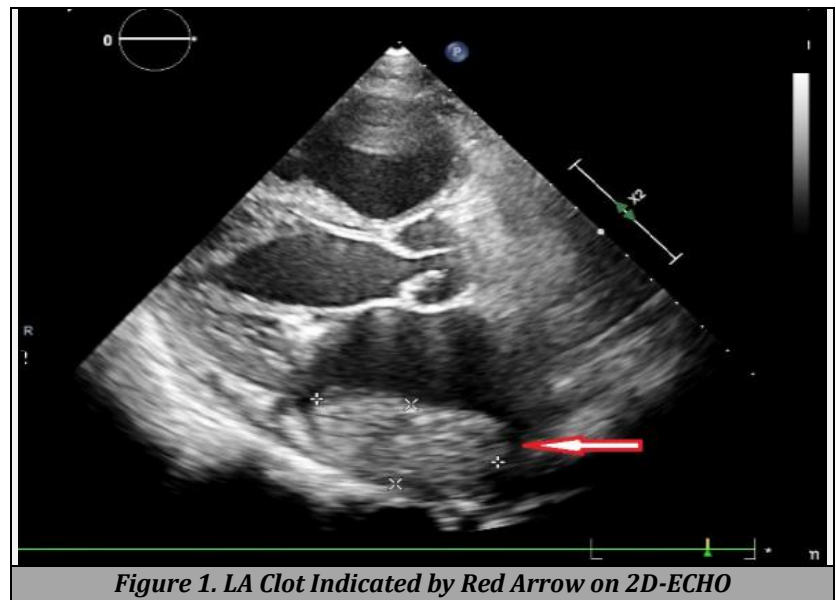

Echocardiography (ECHO) is a vital tool in the diagnostic armamentarium of $\mathrm{AF}$ as it gives valuable information regarding the cardiac function, complications and prognosis in AF.16 Left atrial dilatation indicates chronicity and left ventricular dysfunction, thus resulting in AF. Normally LA size is less than $40 \mathrm{~mm}$, however, size more than $45-50 \mathrm{~mm}$ increases the risk of development of $\mathrm{AF}$ by four-fold and it is observed that sustained AF in turn can further increase the LA size. Thus, LA size is an important predictor of AF as well as its complications such as stroke as $20-30 \%$ of strokes occur as a result of AF. ${ }^{17}$ In the present study $32 \%$ of patients were found to have a LA size of more than $50 \mathrm{~mm}$. In addition, a study by Mahmood ul Hassan et al reported a significant correlation between LA size of greater than $45 \mathrm{~mm}$ and LA clot in AF. ${ }^{18} \mathrm{LA}$ clot was observed in only $4 \%$ of patients in the present study unlike the study by Mohan G et al with $14.5 \%$ of patients exhibiting LA clot. ${ }^{2}$ It is noticed that trans oesophageal ECHO has a greater sensitivity in picking up LA clots in comparison with a transthoracic ECHO. ${ }^{19}$ This lower percentage could be attributed to the fact that all the patients in the present study were subjected to transthoracic ECHO, hence, the presence of LA clot could have been easily missed on transthoracic ECHO.

\section{CONCLUSIONS}

Hypertension is one of the leading causes of $\mathrm{AF}$, accounting for more cases of AF than any other risk factor. In the present study, RHD is the second most common cause, its prevalence reducing owing to the rampant usage of antibiotics. As the prevalence of hypertension is steadily on the rise, it contributes significantly to the cardiovascular risk and death including AF. However, enforcing healthy lifestyle habits, early screening and control of hypertension can in turn reduce the risk of developing AF.

Data sharing statement provided by the authors is available with the full text of this article at jemds.com.

Financial or other competing interests: None.

Disclosure forms provided by the authors are available with the full text of this article at jemds.com.

\section{REFERENCES}

[1] Michaud GF, Stevenson WG. Atrial fibrillation. In: Jameson J, Fauci AS, Kasper DL, et al. eds. Harrison's principles of internal medicine. 20 $0^{\text {th }}$ edn. New York, NY: McGraw-Hill 2018.

[2] Mohan G, Kaur S, Kaur R, et al. The study of clinical and echocardiographic assessment of patients with atrial fibrillation. Int J Adv Med 2019;6(4):1041-5.

[3] Meeran SS, Saravanan MA, Geetha S, et al. A study on clinical profile of patients with atrial fibrillation. IAIM 2018;5(2):105-10.

[4] Allessie MA, Boyden PA, Camm AJ, et al. Pathophysiology and prevention of atrial fibrillation. Circulation 2001;103(5):769-77.

[5] Ott A, Breteler MM, de Bruyne MC, et al. Atrial fibrillation and dementia in a population-based study: the Rotterdam Study. Stroke 1997;28(2):316-21.

[6] Go AS, Hylek EM, Phillips KA, et al. Prevalence of diagnosed atrial fibrillation in adults national implications for rhythm management and stroke prevention: the AnTicoagulation and Risk Factors in Atrial Fibrillation (ATRIA) Study. JAMA 2001;285(18):2370-5. 
[7] Wolf PA, Abbott RD, Kannel WB. Atrial fibrillation as an independent risk factor for stroke: the Framingham Study. Stroke 1991;22(8):983-8.

[8] Lake FR, Cullen KJ, de Klerk NH, et al. Atrial fibrillation and mortality in an elderly population. Aust $\mathrm{N} \mathrm{Z} \mathrm{J} \mathrm{Med}$ 1989;19(4):321-6.

[9] Phillips SJ, Whisnant JP, O'Fallon WM, et al. Prevalence of cardiovascular disease and diabetes mellitus in residents of Rochester, Minnesota. Mayo Clin Proc 1990;65(3):34459.

[10] Furberg CD, Psaty BM, Manolio TA, et al. Prevalence of atrial fibrillation in elderly subjects (the Cardiovascular Health Study). Am J Cardiol 1994;74(3):236-41.

[11] Singh R, Kashyap R, Bhardwaj R, et al. The clinical and etiological profile of atrial fibrillation after echocardiography in a tertiary care centre from North India - a cross sectional observational study. Int J Res Med Sci 2017;5(3):847-50.

[12] Al-Khatib SM, Wilkinson WE, Sanders LL, et al. Observations on the transition from intermittent to permanent atrial fibrillation. American Heart Journal 2000;140(1):142-5.
[13] Benjamin EJ, Levy D, Vaziri SM, et al. Independent risk factors for atrial fibrillation in a population-based cohort. The Framingham Heart Study. JAMA 1994;271(11):8404.

[14] Prystowsky EN, Padanilam BJ, Fogel RI. Treatment of atrial fibrillation. JAMA 2015;314(3):278-88.

[15] Levy S, Maarek M, Coumel P, et al. Characterization of different subsets of atrial fibrillation in general practice in France: the ALFA study: the College of French Cardiologists. Circulation 1999;99(23):3028-35.

[16] Troughton RW, Asher CR, Klein AL. The role of echocardiography in atrial fibrillation and cardioversion. Heart 2003;89(12):1447-54.

[17] Chopra HK, Wander GS, Chandra P, et al. Atrial fibrillation update: a textbook of cardiology. $1^{\text {st }}$ edn. Jaypee Brothers Medical Publishers 2017:262-3.

[18] ul Hassan M, Hussain C, Gul AM, et al. Frequency of left atrial and appendage clot in patients with severe mitral stenosis. J Ayub Med Coll Abbottabad 2010;22(2):40-2.

[19] Kapral MK, Silver FL. Preventive health care, 1999 update: 2. Echocardiography for the detection of a cardiac source of embolus in patients with stroke. Canadian Task Force on Preventive Health Care. CMAJ 1999;161(8):989-96. 